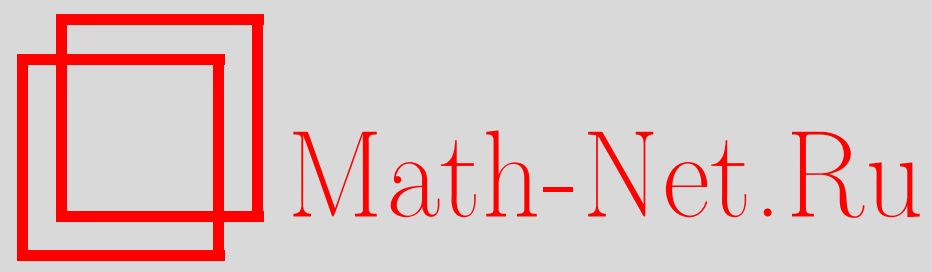

А. А. Карацуба, О нижних оценках максимума модуля $\zeta(s)$ в малых областях критической полосы, Матем. заметки, 2001, том 70, выпуск 5, 796-797

DOI: https://doi.org/10.4213/mzm792

Использование Общероссийского математического портала Math-Net.Ru подразумевает, что вы прочитали и согласны с пользовательским соглашением http://www.mathnet.ru/rus/agreement

Параметры загрузки:

IP : 54.209.52.79

26 апреля 2023 г., 18:30:44

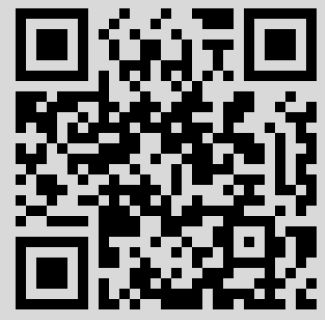




\section{О НИЖНИХ ОЦЕНКАХ МАКСИМУМА МОДУЛЯ $\zeta(s)$ В МАЛЫХ ОБЛАСТЯХ КРИТИЧЕСКОЙ ПОЛОСЫ}

\section{А. А. Карацуба}

В статье автора [1] получены нижние оценки величины

$$
\max _{T \leqslant t \leqslant T+\Delta}|\zeta(\sigma+i t)|
$$

где $\zeta(s)$ - дзета-функция Римана, $\sigma$ - фиксированное число с условием $0.5 \leqslant \sigma \leqslant 1, T \geqslant T_{1}>0$, $\Delta=\Delta(T) \rightarrow 0$ при $T \rightarrow+\infty$.

Здесь рассматривается ослабленньй вариант этой проблемы, а именно, нижние оценки $|\zeta(s)|$ при условии, что $\left|s-s_{1}\right|=\Delta=\Delta\left(s_{1}\right) \rightarrow 0,\left|s_{1}\right| \rightarrow+\infty, 0<\operatorname{Re} s_{1}<1$. На основе теоремы Адамара о трех кругах и простейших оценок $|\zeta(s)|$ получается нижняя оценка соответствующей величины, подобная нижней оценке из [1]. Больший интерес, чем теорема, представляют сформулированные в конце статьи гипотезы, которые подобны гипотезам из [1], но несколько слабее последних.

Пусть, далее, $s=\sigma+i t, s_{1}=\sigma_{1}+i t_{1}$, где $\sigma, \sigma_{1}, t, t_{1}$-вещественные числа, причем $0.5 \leqslant \sigma_{1} \leqslant 1$, $t_{1} \geqslant 4 ; i^{2}=-1 ; 0<\Delta<1 / 3 ; c_{1}, c_{2}, \ldots$ - абсолютные положительные постоянные. Определим функцию $F\left(s_{1} ; \Delta\right)$ равенством

$$
F\left(s_{1} ; \Delta\right)=\max _{\left|s-s_{1}\right|=\Delta}|\zeta(s)| .
$$

Tеорема. Cуществует абсолютная постоянная $A>0$ mакая, ито при $t_{1} \geqslant A$ выполняется следующее неравенство:

$$
F\left(s_{1} ; \Delta\right) \geqslant\left|s_{1}\right|^{6 \log \Delta}
$$

Для доказательства теоремы нужна лемма, известная в теории функций как теорема Адамара о трех кругах.

ЛЕмма. Пусть $0<r_{1}<r_{2}$ и функиия $f(s)$ однозначна и регулярна в области $r_{1} \leqslant$ $|s| \leqslant r_{2} . E c \Omega u$

$$
M(r)=\max _{|s|=r}|f(s)|
$$

то при $r_{1} \leqslant r \leqslant r_{2}$ имеет место оченка

$$
M(r) \leqslant\left(M\left(r_{1}\right)\right)^{\alpha}\left(M\left(r_{2}\right)\right)^{\beta},
$$

əде

$$
\alpha \log \frac{r_{2}}{r_{1}}=\log \frac{r_{2}}{r}, \quad \beta \log \frac{r_{2}}{r_{1}}=\log \frac{r}{r_{1}} .
$$

Доказательство этого утверждения см., например, в [2, с. 456].

ДоКАЗАТЕЛЬСТВо тЕОРЕМЫ. Возьмем в лемме

$$
f(s)=\zeta\left(s+s_{1}\right), \quad r_{1}=\Delta, \quad r=1.5, \quad r_{2}=2 .
$$

Из определения функции $M(r)$ следует, что

$$
M\left(r_{1}\right)=F\left(s_{1} ; \Delta\right), \quad M(r) \geqslant\left|\zeta\left(\sigma+1.5+i t_{1}\right)\right| \geqslant 6 \pi^{-2} .
$$

В свою очередь, $M\left(r_{2}\right)$ не превосходит максимума $|\zeta(\sigma+i t)|$, где $\sigma=\sigma_{1}+2 \cos \varphi, t=t_{1}+2 \sin \varphi$, $0 \leqslant \varphi<2 \pi$. Изусловий на $\sigma_{1}$ и $t_{1}$ получаем неравенства для $\sigma$ и $t:-1.5 \leqslant \sigma \leqslant 3, t_{1}-2 \leqslant t \leqslant t_{1}+2$. 
Полагая $x=\sqrt{t /(2 \pi)}$, для $\zeta(\sigma+i t)$ при $-3 \leqslant \sigma \leqslant 3$ имеем следующее приближенное равенство (приближенное функциональное уравнение Харди-Литтлвуда, см., например, [3, с. 73]):

$$
\zeta(\sigma+i t)=\sum_{n \leqslant x} n^{-\sigma-i t}+h(\sigma ; t) \sum_{n \leqslant x} n^{-1+\sigma+i t}+O\left(t^{-0.5 \sigma}\right),
$$

где

$$
h(\sigma ; t)=\pi^{\sigma-0.5+i t} \Gamma\left(\frac{1-\sigma}{2}-i \frac{t}{2}\right) \Gamma^{-1}\left(\frac{\sigma}{2}+i \frac{t}{2}\right) .
$$

При $t \geqslant t_{1}$ для $\Gamma(\sigma+i t)$ справедлива асимптотическая формула

$$
\Gamma(\sigma+i t)=t^{\sigma-1 / 2+i t} e^{-\pi t / 2-i t+i \pi(\sigma-1 / 2) / 2} \sqrt{2 \pi}\left(1+O\left(t^{-1}\right)\right) .
$$

Поэтому

$$
|h(\sigma ; t)| \leqslant c_{1} t^{1 / 2-\sigma}, \quad|\zeta(\sigma+i t)| \leqslant \begin{cases}c_{2} t^{(1-\sigma) / 2}, & \text { если } \sigma \leqslant 0.9, \\ c_{2} t^{(1-\sigma) / 2} \log t, & \text { если } 0.9 \leqslant \sigma \leqslant 1-(\log t)^{-1}, \\ c_{2} \log t, & \text { если } \sigma>1-(\log t)^{-1} .\end{cases}
$$

Отсюда следует нужная нам верхняя оценка $M\left(r_{2}\right)$ :

$$
M\left(r_{2}\right) \leqslant c_{3}\left|s_{1}\right|^{5 / 4} \text {. }
$$

Так как

$$
\alpha=\frac{\log (4 / 3)}{\log (2 / \Delta)}, \quad \beta=\frac{\log (3 /(2 \Delta))}{\log (2 / \Delta)}
$$

то из (1)-(3) находим

$$
6 \pi^{-2} \leqslant\left(F\left(s_{1} ; \Delta\right)\right)^{\alpha}\left(c_{3}\left|s_{1}\right|^{5 / 4}\right)^{\beta}, \quad F\left(s_{1} ; \Delta\right) \geqslant\left|s_{1}\right|^{6 \log \Delta},
$$

если только $t_{1} \geqslant A>0$, где $A$ - абсолютная постоянная. Теорема доказана.

Каждая следующая из сформулированных гипотез сильнее предыдущей.

ГипотезА 1. Существуют абсолютные постоянные $c>0, A>0$ и функция $\Delta=\Delta\left(s_{1}\right)$, $\Delta\left(s_{1}\right) \rightarrow 0$ при $\left|s_{1}\right| \rightarrow+\infty$, такие, что при $t_{1} \geqslant A$ выполняется оченка

$$
F\left(s_{1} ; \Delta\right) \geqslant\left|s_{1}\right|^{-c} \text {. }
$$

ГИпотеЗА 2. Гипотеза 1 выполняется при

$$
\Delta=\left(\log \log \left|s_{1}\right|\right)^{-1} .
$$

ГиПотезА 3. Гипотеза 1 выполняется при

$$
\Delta=\left(\log \left|s_{1}\right|\right)^{-1}
$$

\section{СПИСОК ЦИТИРОВАННОЙ ЛИТЕРАТУРЫ}

1. Карацуба А. А. // Докл. РАН. 2001. Т. 376. № 1. С. 15-16. 2. Прахар К. Распределение простых чисел. М.: Мир, 1967. 3. Карацуба А. А. Основы аналитической теории чисел. М.: Наука, 1983. 